\title{
COMPONENTE DEMOGRÁFICO DO SISTEMA DE INFORMAÇÃO DA ATENÇÃO À SAÚDE INDÍGENA, DSEI-X̧AVÁNTE, MAŢO GROSSO, BRASIL
}

\author{
Luciene Guimarães de Soura* \\ Ricardo Ventura Santos ${ }^{* *}$
}

\begin{abstract}
Este estudo analisa a consistência do módulo demográfico do Sistema de Informação da Atenção da Saúde Indígena para o Distrito Especial Indígena Xavante (DSEI), Mato Grosso, no período de 1999 a 2004. Os dados foram obtidos através de relatórios disponibilizados pelo SIASIWEB. A base de dados foi investigada com vistas a detectar inconsistências, incluindo mais de um registro para um mesmo evento. Para todo o DSEI, a taxa de mortalidade infantil (TMI) no período passou de 89,2 para 83,8 por mil após as correções na base de dados. Ao se analisar por polo-base, as alterações foram ainda mais substanciais. No caso do Polo-base de Água Boa, a redução da TMI foi de 43,3 para 21,3 por mil. As taxas brutas de mortalidade e de natalidade também experimentaram redução após as correções. Esses achados evidenciam problemas significativos na base de dados sobre saúde do povo Xavante, com a geração de indicadores demográficos que se distanciam da situação real da população. Os autores destacam a necessidade de aprimoramento da coleta e análise dos dados demográficos no âmbito do sistema de informação sobre a saúde indígena.
\end{abstract}

PALAVRAS-CHAVE: Xavante, demografia indígena, sistema de informação, indicadores demográficos, índios sul-americanos.

\section{INTRODUÇÃO}

Em 1999, a responsabilidade pela prestação da assistência à saúde dos povos indígenas passou da Fundação Nacional do Índio (FUNAI) para a Fundação Nacional de Saúde (FUNASA). No novo modelo, concretizou-se a implantação de um serviço de saúde voltado especificamente para os povos indígenas e estruturado segundo divisão territorial em distritos, quais sejam, os chamados Distritos Sanitários Especiais Indígenas ou DSEI, vinculados ao Sistema Único de Saúde (SUS). Atualmente, existem 34 distritos em todo o país (Garnelo; Macedo; Brandão, 2003; Santos et al., 2008).

No âmbito das estratégias de reestruturação do sistema de saúde destinado aos povos indígenas, destaca-se a implantação de um sistema de informação. Segundo a própria FUNASA,

\footnotetext{
* Doutora em Saúde Pública. Antropóloga sanitarista da Fundação Nacional de Saúde (Funasa).

Rua Coelho e Castro, 6. Saúde. $6^{\circ}$ Andar/Assessoria de Saúde Indígena. Cep: 20081-060. Rio de Janeiro - RJ Brasil. lucienegsouza@uol.com.br

* * Doutor em Antropologia. Professor associado do Museu Nacional/UFRJ. Pesquisador titular na Escola Nacional de Saúde Pública/Fiocruz.santos@ensp.fiocruz.br
}

\begin{abstract}
... o acompanhamento e avaliação ...[da política de atenção à saúde indígena] terá como base o Sistema de Informação da Atenção à Saúde Indígena - SIASI. O SIASI deverá subsidiar os órgãos gestores e de controle social quanto à indispensável compatibilidade entre o diagnóstico situacional dos problemas de saúde identificados e as prioridades estabelecidas nos níveis técnico, social e político, visando a coerência entre ações planejadas e efetivamente executadas (2002, p.19).
\end{abstract}

A implantação do Sistema de Informação da Atenção à Saúde Indígena (SIASI) aconteceu a partir de 2000, na forma de módulos, tendo até o presente sido implantados os seguintes módulos: demográfico, morbidade e imunização (Sousa; Scatena; Santos, 2007). Embora as informações devam alimentar continuamente os bancos de dados nacionais, tendo seu acesso garantido a qualquer usuário, e ser o instrumento para a elaboração do planejamento dos DSEIs (Fundação Nacional de Saúde, 2002), permanecem limitações no uso do SIASI. Além do fato de nem todos os módulos terem sido plenamente implantados, o acesso e a operacionalização permanecem restritos. ${ }^{1}$

${ }^{1}$ Para saber mais sobre potenciais e limitações do SIASI, ver Sousa et al., 2007. 
Outros aspectos relevantes na implantação do SIASI dizem respeito às dificuldades técnicas e de pessoal (Sousa; Scatena; Santos, 2007). Por exemplo, houve grandes dificuldades na estruturação de um sistema de alimentação das bases, já que os registros precisavam ser coletados e processados em regiões distantes, com dificuldade de acesso e comunicação (inclusive via computador). Acrescentese a elevada rotatividade dos profissionais que compõem as equipes de saúde indígena, o que dificulta a padronização na coleta e a qualidade dos dados epidemiológicos e demográficos.

O presente estudo tem por objetivo verificar, a partir da base nacional do SIASI, a consistência dos dados demográficos e o seu impacto nas taxas brutas de natalidade, mortalidade e mortalidade infantil.É utilizada, como estudo de caso, a base do SIASI referente ao Distrito Especial Indígena Xavante - DSEI -, Mato Grosso, no período de 1999 a 2004. Os resultados trazem subsídios relevantes para o aprimoramento do sistema.

\section{FONTES DE DADOS E MÉTODOS}

Os dados demográficos analisados neste trabalho foram obtidos a partir de relatórios disponibilizados eletronicamente pelo SIASIWEB (www.funasa.gov.br/siasi) para o DSEI, Mato Grosso, com foco no chamado Módulo Demográfico.

A pesquisa é relativa aos dados referentes ao período entre 1999 e 2004. O banco de dados foi acessado no dia 31 de janeiro de 2006. As variáveis individuais selecionadas para análise foram: nome, sexo, data de nascimento, data de óbito, filiação (nome do pai e da mãe), aldeia e Polo-Base. No caso do DSEI Xavante, há quatro polos-base (Água Boa, Campinápolis, Paranatinga e São Marcos), que são as subunidades nas quais se estruturam os distritos.

A saída (output) dos dados disponibilizados pela FUNASA tem o formato de planilhas do Excel. As análises aqui apresentadas foram também conduzidas com esse aplicativo.

A identificação de registros repetidos seguiu as fases: pré-processamento dos dados; identificação de registros pareados (matches); verificação de registros pareados que se referiam ao mesmo indivíduo.

Durante a fase de pré-processamento da base de dados, foram identificados 13.616 indivíduos. Foram feitas depurações a partir das variáveis "data de óbito" e "data de nascimento". Constatou-se que permaneciam na base 19 indivíduos cujos óbitos haviam ocorrido entre 1990 e 1998, e 31 óbitos registrados para o ano de 2005. A data do último registro de nascimento era 30 de abril de 2005. Foram eliminados os óbitos do período anterior a 1999. Optou-se também por descartar os 31 óbitos e os 145 registros de nascimento do ano de 2005, visando a trabalhar com dados de anos completos. Nessa etapa, também foram corrigidos os erros evidentes de digitação de data de nascimento, tais como, data de nascimento posterior à data de entrada dos dados.

Para fins de pareamento, foram analisadas comparativamente as variáveis individuais combinadas segundo diferentes arranjos: 1) nome, sexo, data de nascimento, data de óbito, nome do pai, nome da mãe, aldeia e Polo-Base; 2) data de nascimento, data de óbito, nome, sexo, nome do pai, nome da mãe, aldeia e Polo-Base; 3) nome da mãe, nome do pai, data de nascimento, nome, data do óbito, sexo, aldeia e Polo-Base. O critério para identificação de duplicidade de registro considerou cinco variáveis: nome, sexo, data de nascimento, nome da mãe, nome do pai. Dentre essas cinco, pelo menos três precisam coincidir integralmente para que se considere a existência de uma duplicidade.

Ainda com relação ao critério para identificação de duplicidade, foram consideradas quatro categorias: duplicidade vivo com vivo - indivíduo registrado duas vezes, apresentando duas datas de nascimento; duplicidade de óbito com óbito - indivíduos registrados duas vezes, apresentando duas datas de óbito; duplicidade de vivo com óbito - indivíduos registrados duas vezes, sem e com data de óbito; óbito triplicado - indivíduos registrados três vezes, apresentando três datas de óbito.

Após a classificação, os registros repetidos foram excluídos, permanecendo os registros com 
mais campos de variáveis preenchidas.

As taxas brutas de natalidade e de mortalidade foram calculadas utilizando-se o número total de nascimentos e de óbitos no período de 19992004, dividido pela população residente da área no mesmo período considerado e multiplicado por mil. Como não foi possível desmembrar a população total por ano de referência, dividiu-se o resultado final pelo número de anos analisados. A taxa de mortalidade infantil foi calculada pelo método direto, ou seja, o número de óbitos de menores de 1 ano de idade foi dividido pelo número de nascidos vivos no mesmo período e multiplicado por mil.

\section{RESULTADOS}

Já na fase do pré-processamento foram observados problemas na atualização dos dados vitais na base do SIASI, tendo-se verificado que o último registro de nascimento aconteceu em abril de 2005. A falta de definição da população no final de cada ano, fundamental para o cálculo dos indicadores segundo o ano, foi outro problema identificado. Ou seja, não é possível saber de forma direta qual é o montante populacional por polobase ano a ano, o que impossibilitou o cálculo das taxas por ano de referência, levando-nos a efetuar a análise de período.

Foram observadas diferenças importantes na qualidade de preenchimento dos dados entre os polos-base. O Polo-Base de Água Boa foi o que apresentou maior número de registros duplicados, contribuindo com $75 \%$ de todas as duplicidades (Tabela 1). Nesse mesmo Polo, também foi eviden- ciada a possível ocorrência de sub-registro de óbitos, já que não foram registrados óbitos para os anos de 1999 e 2000. Os óbitos gerais, sem natimorto e aborto, passaram a ser registrados somente em 2001 (3 óbitos). Em 2002, foram 11 óbitos nesse Polo-Base, com maior volume em 2003 (28 óbitos) e 2004 (34 óbitos).

As taxas de mortalidade geral observadas para o Polo-Base de Água Boa são também compatíveis com a hipótese de subnotificação de óbitos. Em geral, os valores da taxa bruta de mortalidade das populações oscilam, em maior parte, entre seis e 12 óbitos por mil habitantes (OPAS/OMS, 2000). Para o Brasil, tem sido considerado aceitável pelo Ministério da Saúde um coeficiente de 6,0 por mil habitantes (BRASIL, 1992). Abaixo desses valores, deve-se considerar a possibilidade de sub-registro ou má qualidade dos dados. As taxas brutas de mortalidade observadas no Polo-Base de Água Boa, com correção e sem correção, ficaram abaixo de 5,0 por mil habitantes, possivelmente apontando problemas com a base de dados.

Nos quatro Polos-Base do DSEI Xavante foram identificados registros indevidos de abortos (9) e natimortos (42) como nascidos vivos, ou seja, com data de nascimento e data de óbito. Houve também um caso de registro em duplicidade entre um natimorto e um nascido vivo. Quanto ao número de registros duplicados de óbitos, foram detectadas 39 duplicidades de óbito com óbito, 10 duplicidades entre óbito com vivo e 2 óbitos triplicados (Tabela 1).

As diferenças de grafia, abreviações e mesmo erro de digitação introduziram variações que fizeram com que dois registros de uma mesma pes-

Tabela 1 - Erros mais frequentes, segundo Pólo-Base, SIASI/ DSEI Xavante, Mato Grosso, 1999-2004

\begin{tabular}{l|c|c|c|c|c|c|c}
\hline Pólo-Base & $\begin{array}{c}\text { Duplicidade- } \\
\text { Vivo/vivo }\end{array}$ & $\begin{array}{c}\text { Erro digitação } \\
\text { da data de } \\
\text { nascimento }\end{array}$ & Aborto* & Natimorto* & $\begin{array}{c}\text { Duplicidade } \\
\text { óbito/ óbito }\end{array}$ & $\begin{array}{c}\text { Duplicidade } \\
\text { óbito/vivo } \\
\text { Triplicado }\end{array}$ \\
\hline Água Boa & 604 & - & 5 & 4 & 18 & 1 & - \\
\hline Campinápolis & 93 & 48 & 3 & 23 & 8 & 5 & - \\
\hline Paranatinga & 29 & 6 & - & 2 & 2 & - & - \\
\hline São Marcos & 84 & 35 & 1 & 13 & 42 & 39 & 10 \\
\hline DSEI & 810 & 89 & 9 & 4 & 2 \\
\hline
\end{tabular}

* Referem-se a aborto e natimorto registrados como nascidos vivos 
soa não correspondessem integralmente um ao outro. Foram também observados 89 erros de digitação com data de nascimento posterior à data de entrada dos dados (por exemplo, ano de nascimento 2053, 2048). Esses erros eram referentes a indivíduos adultos e foram corrigidos.

Observou-se que a ocorrência de duplicidade no registro de crianças menores de 5 anos na base de dados em geral deriva de um erro recorrente. A
SIASI, no período entre 1999 e 2004, a população do Distrito Especial Indígena Xavante era de 13.421 e 12.601 indivíduos, sem e com correções, respectivamente. Em relação aos indicadores, verificou-se que, com exceção da taxa bruta de mortalidade e mortalidade infantil para o Polo-Base de Paranatinga e da mortalidade infantil para Campinápolis, os demais apresentaram declínio após as correções (Tabela 2).

Tabela 2 - Taxa Bruta de Natalidade, Taxa Bruta de Mortalidade e Mortalidade Infantil (por mil), dados sem e com correçáo, segundo Pólo-Base, SIASI/DSEI Xavante, Mato Grosso, 1999-2004

\begin{tabular}{|c|c|c|c|c|c|c|c|}
\hline \multicolumn{8}{|c|}{ Sem correção } \\
\hline Pólo-Base & População & NV & $\begin{array}{l}\text { Óbitos } \\
\text { gerais }^{1}\end{array}$ & $\begin{array}{l}\text { Óbitos } \\
<1 \text { ano }^{1}\end{array}$ & TBN & TBM & TMI \\
\hline Água Boa & 3447 & 1016 & 85 & 44 & 58,9 & 4,9 & 43,3 \\
\hline Campinápolis & 5196 & 1510 & 337 & 160 & 58,1 & 13,0 & 106,0 \\
\hline Paranatinga & 1949 & 490 & 95 & 42 & 50,3 & 9,7 & 85,7 \\
\hline São Marcos & 2829 & 786 & 184 & 93 & 55,6 & 13,0 & 118,3 \\
\hline DSEI & 13421 & 3802 & 701 & 339 & 56,7 & 10,4 & 89,2 \\
\hline \multicolumn{8}{|c|}{ Com correção } \\
\hline Pólo-Base & População & NV & $\begin{array}{l}\text { Óbitos } \\
\text { gerais }^{2}\end{array}$ & $\begin{array}{c}\text { Óbitos } \\
<1 \text { ano }^{2}\end{array}$ & TBN & TBM & TMI \\
\hline Água Boa & 2842 & 797 & 45 & 17 & 56,1 & 3,2 & 21,3 \\
\hline Campinápolis & 5098 & 1.130 & 316 & 124 & 44,3 & 12,4 & 109,7 \\
\hline Paranatinga & 1920 & 414 & 98 & 36 & 43,1 & 10,2 & 87,0 \\
\hline São Marcos & 2741 & 618 & 155 & 71 & 45,1 & 11,3 & 114,9 \\
\hline DSEI & 12601 & 2959 & 614 & 248 & 47,0 & 9,7 & 83,8 \\
\hline
\end{tabular}

${ }^{1}$ Com aborto, natimorto e duplicidade.

2 Óbitos sem aborto, natimorto e duplicidade.

análise dos registros sugere que, de forma sistemática, quando a criança nasce, ela é registrada como recém nascido de "uma dada mulher". Não obstante, quando recebe um nome, é novamente registrada sem que o digitador observe que, naquela família, já existe uma criança com mesmo sexo, data de nascimento e filiação.

Foram detectados problemas nos registros das datas de nascimento por filiação (mãe e pai). Havia, na base de dados, registros de indivíduos com a mesma filiação (especificamente mãe), mas com nome e data de nascimento diferentes. Isto fez com que, por exemplo, o espaçamento interpartal, em alguns casos, fosse de menos de 6 meses. Entretanto, esses erros não podem ser explicados pela presença de registros repetidos no banco de dados. Devido a isso, não foram corrigidos.

Na base de dados de âmbito nacional do
As taxas de mortalidade infantil para todos os polo-base apresentaram diferenças entre $15 \mathrm{a}$ 25 pontos percentuais após as correções. Chama atenção o declínio para o Polo-Base de Água Boa, que passou de 43,3 para 21,3 por mil nascidos vivos, ou seja, uma redução de 50,8 por cento após as correções (Tabela 3).

Tabela 3 - Diferença percentual entre os indicadores antes e após correções, segundo Pólo-Base, SIASI/DSEI Xavante, Mato Grosso. 1999-2004

\begin{tabular}{l|c|c|c}
\hline \multicolumn{4}{c}{ Diferença (\%) } \\
\hline Pólo-Base & TBN & TBM & TMI \\
\hline Água Boa & $-5,0$ & $-53,1$ & $-50,8$ \\
\hline Campinápolis & $-31,2$ & $-4,8$ & $+3,4$ \\
\hline Paranatinga & $-16,7$ & $+4,9$ & $+1,5$ \\
\hline São Marcos & $-23,3$ & $-15,0$ & $-3,0$ \\
\hline DSEI & $-20,6$ & $-7,2$ & $-6,4$ \\
\hline
\end{tabular}




\section{COMENTÁRIOS FINAIS}

Uma vez que variáveis demográficas são comumente utilizadas na caracterização da saúde de populações, a falta de dados de qualidade repercute diretamente na gestão e avaliação das políticas públicas. Muitos dos principais indicadores de saúde são indicadores demográficos e dependentes de base populacional, como o coeficiente de mortalidade infantil, a esperança de vida ao nascer, as taxas brutas e específicas de mortalidade, dentre outros.

Inquestionavelmente, a concepção, o desenvolvimento e a implantação de um sistema de informação voltado para a saúde indígena, como o SIASI, representam um inegável avanço, além de ser de vital importância em diversas esferas (Sousa; Scatena; Santos, 2007). Segundo esses autores, apesar de inúmeras limitações, tanto na concepção quanto na operacionalização, o SIASI apresenta potencialidades. Dentre elas, destaca-se a integralidade, ou seja, o fato de se propor a agregar, em um único sistema, uma multiplicidade de informações importantes para o conhecimento da realidade de saúde dos povos indígenas.

A discussão de aspectos eminentemente técnicos, contudo, não deve nos deixar perder de vista que a função de um sistema de informação é a disponibilidade de informações de qualidade, onde e quando necessárias. Para alcançar esse objetivo, deve-se manter um rígido controle de qualidade em todos os elementos e fases que compõem o sistema de informação. De acordo com Sanches et al.:

... nenhuma informação terá melhor qualidade que os dados que serviram de base para a sua elaboração. Se quem gera o dado não percebe o sentido ou a necessidade de seu registro, e esta atividade se torna apenas uma rotina burocrática, pode-se esperar que a qualidade dos dados alimentados no sistema decaia; por outro lado, se existe um retorno, sob a forma de divulgação ampla de indicadores derivados destes dados de um modo que a necessidade de seu registro fique clara para quem o gera, é razoável esperar um maior comprometimento e cuidado na sua captação, digitação e retroalimentação. Por sua vez isto também facilitará o processo de crítica das variáveis, minimizando as inconsistências nas bases de dados (2003, p.345).
Os resultados aqui apresentados demonstram importantes fragilidades do sistema de informação, o que potencialmente traz consequências para o funcionamento do modelo de atenção. Dado que o SIASI deve conter informações capazes de favorecer a construção de indicadores que possam subsidiar a avaliação da situação de saúde e da organização do serviço de saúde nos DSEIs, quanto ao acesso, cobertura e efetividade, a análise dos indicadores produzidos no âmbito dos distritos é de fundamental importância e deveria se constituir em uma fase obrigatória dentro do próprio sistema. Após quase 8 anos de sua implantação, o SIASI, por razões diversas, ainda não é capaz de gerar relatórios minimamente satisfatórios e informações confiáveis para o planejamento e avaliação das ações de saúde (Sousa; Scatena; Santos, 2007).

Tendo em vista o objetivo inicial deste trabalho, que foi a análise do impacto da inconsistência nos indicadores demográficos gerados pelo sistema, observamos que há importantes limitações no que tange à utilização das informações derivadas do banco/SIASI do DSEI Xavante. Dentre outros aspectos, a defasagem temporal de alimentação do sistema e a subenumeração prejudicam a qualidade dos dados alimentados e sugerem a baixa confiabilidade dos indicadores gerados pelo sistema de informação. A análise da mortalidade infantil demonstrou que a possibilidade de registro de abortos e natimortos como nascidos vivos gera tanto um aumento do número de nascidos vivos quanto de óbitos neonatais precoces, levando a distorção e, principalmente, a grandes variações nas taxas de mortalidade infantil. Desse modo, as mudanças nos valores dos indicadores de mortalidade apresentados nesta análise, em geral para patamares menores, não podem ser vistas como uma melhora real das condições de saúde dos Xavante, mas sim um demonstrativo da má qualidade da informação.

No momento da coleta e digitação dos dados Xavante no SIASI, os problemas mais comuns que ocorreram foram subenumeração, superenumeração e registro errôneo de sexo e idade. Há estratégias que podem ser facilmente implementadas para 
enfrentar esses problemas. Normalmente, erros deste tipo podem ser detectados através de atualizações executadas periodicamente. Além desse procedimento, existe a possibilidade de comparações com outros dados existentes (por exemplo, os registros dos nascimentos e óbitos e os registros do acompanhamento do pré-natal, dentre outros). Ainda no momento da digitação, a utilização de máscaras de edição pode evitar que dados com formato incorreto sejam digitados. Por exemplo, é impossível uma data de nascimento posterior à data de entrada dos dados, e esse tipo de inconsistência pode ser verificado pela própria rotina de entrada de dados. Embora o sistema de processamento eletrônico dos formulários conte com algumas rotinas de tratamento de erros (no caso específico do SIASI foram desenvolvidos filtros para evitar duplicidade de registro), verificase que, na prática, esses filtros não estão funcionando adequadamente e devem ser revistos.

Quanto a outras sugestões para enfrentar os problemas identificados, pode-se considerar a possibilidade de constituir, no âmbito do DSEI, uma equipe de técnicos com a função de acompanhar e avaliar rotineiramente a consistência dos dados produzidos em serviço e definir as estratégias de correção e utilização dos mesmos. Essa equipe poderia atuar no nível central do DSEI, possivelmente na sede, acompanhando e analisando comparativamente os dados dos diversos polos. A revisão periódica das informações produzidas pelo DSEI à luz de medidas e indicadores gerados pelo próprio sistema de informação é recomendada para detecção de inconsistências. Se bem conduzidas, essas avaliações poderão sinalizar o momento apropriado para uma nova revisão in loco dos dados de população (por exemplo, a realização de uma contagem anual). De forma contínua e sistemática, uma iniciativa como essa poderia subsidiar (e valorizar) as ações das equipes de saúde que trabalham em campo, indicando eventuais problemas na coleta de dados.

Em conclusão, para melhor conhecer a realidade de saúde e fornecer subsídios para o planejamento, gestão e avaliação das ações de saúde, é fundamental que os sistemas de registro e coleta de dados demográficos acerca dos povos indígenas no Brasil sejam aprimorados. A partir de um estudo de caso, este estudo indica que, apesar de sua fundamental importância, o SIASI, como componente essencial para a organização dos DSEI, não vem cumprindo o papel esperado de fornecer informações confiáveis. Além da reformulação do sistema de informação no âmbito central, faz-se necessário também, maior atenção para as rotinas de coleta e sistematização dos dados no âmbito local.

(Recebido para publicação em julho de 2009) (Aceito em setembro de 2009)

\section{REFERÊNCIAS}

BRASIL. Ministério da Saúde. Estatísticas de mortalidade, Brasil, 1988. Brasília,DF: Centro de Documentação do Ministério da Saúde, 1992.

FUNDAÇÃO NACIONAL DE SAÚDE. (FUNASA) Disponível em: www.funasa. gov.br Acesso em: 14 mar. 2007. Política nacional de atenção à saúde dos povos indígenas. 2.ed. Brasília,DF: Ministério da Saúde, 2002.

GARNELO, L; MACEDO, G; BRANDÃO, L.C. Os povos indígenas e a construção da política de saúde no Brasil. Brasília: OPAS, 2003

ORGANIZACÃO PANAMERICANA DE SAÚDE (OPAS). $126^{a}$ Sessão do Comitê Executivo: funções essenciais de saúde pública. Washington, DC, 2000.

SANCHES, K.R.B. et al. Sistemas de informação em saúde. In: MEDRONHO; R.A. et al. (Org). Epidemiologia. São Paulo: Ed. Atheneu, 2003. p.337-359.

SANTOS, R.V. et al. Saúde dos povos indígenas e políticas públicas no Brasil. In: GIOVANELLA, L. et al. (Org.) Políticas e sistema de saúde no Brasil. Rio de Janeiro: Ed. Fiocruz; Cebes, 2008. p.1035-1056.

SOUSA, M.C; SCATENA, J.H.G; SANTOS R.V. O sistema de informação da atenção à saúde indígena (SIASI): criacão, estrutura e funcionamento. Cadernos de Saúde Pública, Rio de Janeiro, n.23, v.44, p.853-861, 2007. 


\section{DEMOGRAPHIC COMPONENT OF THE INFORMATION SYSTEM OF THE ATTENTION TO INDIGENOUS HEALTH, DSEI-XAVÁNTE, MATO GROSSO, BRAZIL}

\section{Luciene Guimarães de Souza Ricardo Ventura Santos}

This study analyzes the consistence of the demographic module of the System of Information of Attention to Indigenous Health for the Shavante Indian Indigenous Special District (in Portuguese, DSEI), Mato Grosso, from 1999 to 2004. The data were obtained through reports made available by SIASIWEB. The database was investigated trying to detect inconsistencies, including more than a single registration for the same event. For the whole DSEI, the infant mortality rate (in Portuguese, TMI) in the period went from 89,2 to 83,8 per thousand after the corrections in the database. Analyzing by base headquarters, the alterations were even more substantial. In the case of the Água Boa base headquarters, the reduction of TMI went from 43,3 to 21,3 per thousand. The gross mortality and birth rates also experienced reduction after the corrections. Those discoveries evidence significant problems in the database on the health of the Shavante people, generating demographic indicators that are distant of the real situation of the population. The authors point out the need of enhancement of the collection and analysis of the demographic data in the extent of the system of information about the indigenous health.

KeYwords: Shavante Indian, indigenous demography, information system, demographic indicators, South American Indians.
COMPOSANTE DEMOGRAPHIQUE DU SYSTEME D'INFORMATION A L'ATTENTION DE LA SANTE INDIGENE, DSEI-XAVÁNTE, MATO GROSSO, BRESIL

\section{Luciene Guimarães de Souza Ricardo Ventura Santos}

Cette étude analyse la consistance du module démographique du Système d'Information et d'Attention attribué à la Santé Indigène dans le District Spécial Indigène Xavante (Sistema de Informação da Atenção da Saúde Indígena para o Distrito Especial Indígena Xavante - DSEI), dans le Mato Grosso, de 1999 à 2004. Les données ont été obtenues à partir des rapports fournis par le SIASIWEB. La base des données a été étudiée afin d'y détecter les incohérences, y compris le fait d'avoir divers rapports pour un même événement. Dans tout le DSEI, le taux de mortalité infantile (TMI) de cette période, est passé de 89,2 à 83,8 pour mille, après qu'on ait corrigé la base de données. Si l'on analyse par pôle de base, les changements ont été encore plus substantiels. Dans le cas du Pôle de base de Agua-Boa, la réduction du TMI est passée de 43,3 à 21,3 pour mille. Les taux bruts de mortalité et de natalité ont également été réduits après corrections. Ces résultats mettent en évidence d'importants problèmes concernant la base des données relatives à la santé du peuple Xavante, avec une production d'indicateurs démographiques qui s'éloignent de la situation réelle de la population. Les auteurs soulignent le besoin d'améliorer la collecte et l'analyse des données démographiques au sein du système d'information sur la santé des indigènes.

Mots-CLÉs: Xavante, démographie indigène, système d'information, indicateurs démographiques, indiens sud-américains.

Luciene Guimarães de Souza - Doutora em Saúde Pública pela Fundação Oswaldo Cruz. Antropóloga da Fundação Nacional de Saúde (Funasa) e orientadora do Curso de Especialização em Vigilância Alimentar e Nutricional para a Saúde Indígena, modalidade à distância, oferecido pela parceria Fiocruz e Funasa. Desenvolve principalmente os seguintes temas: demografia antropológica; saúde das populações indígenas; epidemiologia e antropologia da saúde; indicadores básicos de saúde.

Ricardo Ventura Santos - Doutor em Antropologia pela Indiana University. Pós-doutor pela University of Massachusetts e MIT. Professor associado do Museu Nacional/UFRJ. Pesquisador titular na Escola Nacional de Saúde Pública/Fiocruz. Além de vários artigos em periódicos nacionais e estrangeiros, publicou "The xavante in transition: health, demography and bioanthropology" (2002), "Epidemiologia e saúde dos povos indígenas no Brasil" (2003), "Demografia dos povos indígenas no Brasil" (2005). Interesses de pesquisa e docência: antropologia biológica; epidemiologia e antropologia da saúde; saúde dos povos indígenas. 
